\title{
Laparoscopic supracervical hysterectomy with the use of the More-Cell-Safe system in a patient with uterine leiomyomas
}

\author{
Paulina Szymczak, Sambor Sawicki, Dariusz Wydra
}

Department of Gynecology, Oncologic Gynecology and Gynecological Endocrinology, Medical University of Gdansk, Poland

\section{INTRODUCTION}

Laparoscopic power morcellation in gynecologic surgery is commonly used to facilitate the removal of a large uterus or uterine fibroids.

In 2014, the U.S. Food and Drug Administration (FDA) and Health Canada released a safety communication, advising against the use of power morcellation due to the risk of malignant dissemination, particularly uterine sarcoma.

The risk of an unexpected uterine sarcoma following surgery for presumed uterine leiomyoma has been estimated at approximately $1 / 350$, and is age-dependent.

\section{CASE REPORT}

A 44-year-old woman was referred to our clinic due to myomatous uterus.

She presented preoperatively with lower abdominal pain and abnormal, painful menstrual bleeding (menarche at age 13 , two births including one cesarean section, BMI - 26), without family history of cancer. Hemoglobin on first day of hospitalization was $12.1 \mathrm{~g} / \mathrm{dL}$ (postoperatively $10.6 \mathrm{~g} / \mathrm{dL}$ ), the C-reactive protein was $1.45 \mathrm{mg} / \mathrm{dL}$.

A transvaginal ultrasound revealed the anteroposterior (AP) diameter of the uterus to be $60 \mathrm{~mm}$, and 4 uterine fibroids in the anterior wall on the right side, and 1 fibroid on the posterior wall. Endometrial thickness was normal, there were no pathologic structures within the adnexa and no free fluid intra-abdominally.

The patient underwent a laparoscopic supracervical hysterectomy with bilateral salpingectomy with the use of the More-Cell-Safe system. The More-Cell-Safe system can be used only with a $10 \mathrm{~mm}$ optic, blunt laparoscopic $12 \mathrm{~mm}$ trocar (Blade Trocar, VAXCON), and a 12-15 mm blunt morcellator.

After amputation of the corpus uteri with both fallopian tubes, an endoscopic bag was inserted into the abdominal cavity using the $12 \mathrm{~mm}$ trocar through a single incision in the suprapubic area. After removing the protective foil, the uterus was placed in the bag and the threads on its edges were pulled from outside the abdominal cavity through the $12 \mathrm{~mm}$ trocar (Fig. 1). Next, the optic-access of the More-Cell Bag was guided externally through the optic trocar. The optic (protected with special disposable sleeve) was inserted through the trocar into the optic-access of the More-Cell Bag, then the More-Cell Bag was insufflated with $\mathrm{CO}^{2}$ (Fig. 2). The power morcellator was inserted via the $12 \mathrm{~mm}$ trocar and the specimen was carefully morcellated inside the bag (Fig. 3). After the complete morcellation, the optic-access of the More-Cell Bag was closed by two tied knots (Fig. 4). The bag was carefully, without applying force, removed from the abdominal cavity through the suprapubic incision (without any damage). The cervical stump was sutured with continuous seam and the left round ligament was attached to it. A drainage tube was placed into the recto-uterine pouch.

The length of the surgery was 161 minutes. The postoperative course was uneventful. The patient was discharged on day 3 of hospitalization. The final histopathological examination confirmed uterine fibroids. 


\section{DISCUSSION}

The statement of the FDA has encouraged scientists to look for new procedures which would allow to morcellate in a safer way, decreasing the risk of unsuspected malignant dissemination. The More-Cell-Safe system could be an acceptable and feasible alternative form of tissue delivery during laparoscopic procedures.

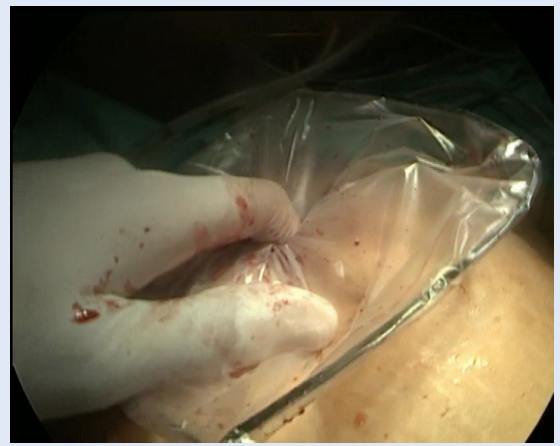

Figure 1. A part of the isolation bag removed through the suprapubic incision

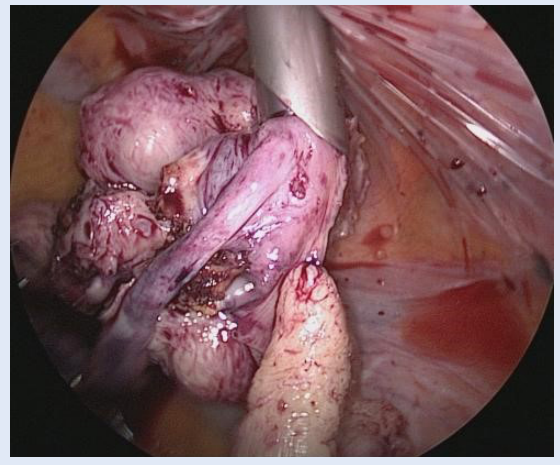

Figure 3. In-bag morcellation process after insufflation with $\mathrm{CO}^{2}$

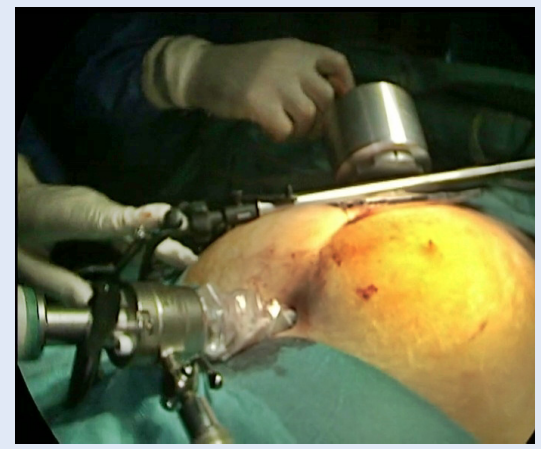

Figure 2. A view of the position of the optic and the morcellator

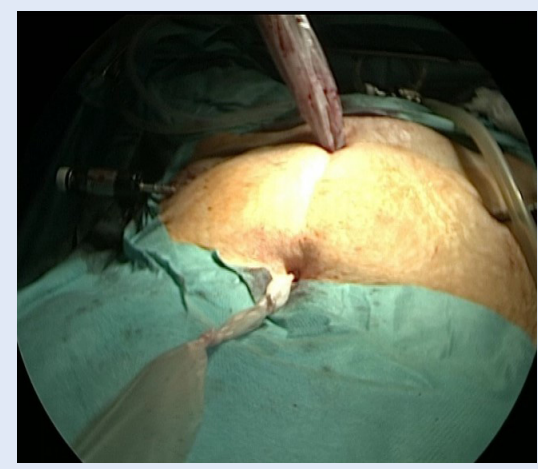

Figure 4. A More-Cell Bag closed by two tied knots 